\title{
Bioinformatic Analysis of IncRNA Mediated Competitive Endogenous RNA Network in Intestinal Ischemia/reperfusion Injury
}

\section{Lin Zhu}

Southern Medical University

\section{Xiao Yang}

Southern Medical University

\section{Zhiwen Yao}

Southern Medical University

\section{Ziyi Wang}

Southern Medical University

\section{Yupei Lai}

Southern Medical University

\section{Shiting Xu}

Southern Medical University

Kexuan Liu

Southern Medical University

Bingcheng Zhao ( $\nabla$ zhaobch@mail2.sysu.edu.cn )

Southern Medical University https://orcid.org/0000-0002-5671-4999

\section{Research}

Keywords: bioinformatics, competitive endogenous RNA, long non-coding RNA, intestinal ischemia/reperfusion injury, miRNA

Posted Date: October 1st, 2021

DOI: https://doi.org/10.21203/rs.3.rs-934215/v1

License: (c) (i) This work is licensed under a Creative Commons Attribution 4.0 International License. Read Full License 


\section{Abstract}

\section{Background}

Recently, an increasing number of studies have reported the roles of competitive endogenous RNA (ceRNA) networks in ischemia/reperfusion (I/R) injury, which include the liver, kidney, heart, brain, and intestine. However, the functions and mechanisms of long non-coding RNAs (IncRNAs), which serve as ceRNA networks in intestinal I/R injury, are still unclear.

\section{Methods}

In this study, bioinformatics methods were used to filter and construct the IncRNA-miRNA-mRNA networks in intestinal I/R injury. RNA expression data were retrieved from NCBI GEO datasets, the expression profiles between mouse small intestine with superior mesenteric artery occlusion and Sham operation was analyzed, and 189 microRNA differential expressed genes(miDEGs) were discovered successfully from miRNA GEO dataset (GSE83701). Next, targeted IncRNAs and mRNA in the database were matched based on miDEGs. Then, IncRNA-miRNA-mRNA networks were constructed with Cytoscape. The hub IncRNA-miRNA-mRNA networks were selected via Cytoscape plug-in CytoHubba and intersected mRNAs of datasets GSE96733 and GSE83701.

Finally, the vital nodes of the ceRNA networks were validated by qPCR.

\section{Results}

The $1700020114 \mathrm{Rik} / \mathrm{mmu}-\mathrm{miR}-7 \mathrm{a}-5 \mathrm{p} / \mathrm{Klf} 4$ axis was postulated to play a potential role in intestinal I/R injury.

\section{Conclusion}

The results shed novel insight into the molecular mechanism of ceRNA networks in intestinal I/R injury and highlighted the potential of $170002700020114 \mathrm{Rik} / \mathrm{mmu}-\mathrm{miR}-7 \mathrm{a}-5 \mathrm{p} / \mathrm{KIf} 4$ ceRNA network in the prevention and treatment of intestinal I/R injury.

\section{Introduction}

I/R-induced organ injury is one of the leading causes of perioperative death worldwide[1]. Among the numerous I/R injuries, intestinal I/R injury is the most difficult to diagnose and treat. It could induce multiorgan function failure, even death, making it one of the major and demanding challenges in medical research. Intestinal I/R injury can result in subsequent aberrant regulation of oxidative stress apoptosis, mitochondrial dysfunction, autophagic pathways, and signal transducers activation[1]. However, the precise molecular mechanism of intestinal I/R injury is still unclear. In order to effectively reduce the damage of intestinal I/R injury and limit the multiple organ injuries caused by intestinal I/R injury, the 
development of innovative treatment and in-depth study is urgently required. Non-coding RNA, including IncRNAs and microRNAs (miRNAs), have been shown to influence I/R injury recently[2].

LncRNA is a non-coding RNA with a length greater than 200 nucleotides which are not translated into a protein[3]. LncRNA has been shown to control gene expression by chromatin modification, transcriptional and post-transcriptional modifications[3]. MicroRNA(miRNA) is a small non-coding RNA with a length between 22-25 nucleotides, which can bind to target RNA transcripts resulting in the repression of mRNA expression or degradation of mRNA.

According to the ceRNA hypothesis proposed by Salmena et al. [4], IncRNAs and mRNAs may share miRNA response elements (MREs). There is growing evidence that IncRNA, mRNA, and circRNA could compete with miRNAs by sharing MREs[5], implying that IncRNAs operate as a sponge for miRNAs by binding with MREs, therefore suppressing the effect of miRNAs on their putative targets. Meanwhile, certain miRNAs can bind with the 3'UTR of IncRNAs or mRNA, causing degradation or suppressing the mRNA production.

The role of miRNAs in the I/R injury has been assessed in several tissues, including the intestine, liver, kidney, heart, and brain[2]. The molecular mechanism of I/R damage is expected to be elucidated by understanding IncRNA-miRNA interactions[2].

A growing number of studies have demonstrated the role of IncRNAs as a ceRNA in the occurrence and progression of I/R injury, including heart, liver, intestinal, and brain injury [5-7]. Xu et al.[7] found and validated that maladjusted IncRNAs were appeared in the early stage of the intestinal I/R injury and were associated with apoptosis. Feng et al.[8] elucidated that circle RNA PRKCB acts as a ceRNA and plays an essential role in intestinal I/R injury via modulating oxidative stress. However, the functions and mechanisms of most IncRNAs act as a ceRNA network in intestinal I/R injury are not well clarified. In this study, IncRNA mediated ceRNA networks related to the intestinal I/R injury were constructed by a set of analysis (Fig. 1).

\section{Results}

\section{Differentially expressed miRNAs}

GSE83701 yielded a total of 1193 miRNAs after preprocessing. A total of 189 miDEGs (107 up-regulated and 82 down-regulated) were obtained (Table 2). Principal component analysis (PCA) indicates that the sets of samples are independent of each other. The volcano plot in Fig. 2B demonstrates that there are two groups of differentially expressed miRNAs. The heatmap (Fig. $2 \mathrm{C}$ ) revealed that differentially expressed miRNAs clearly separated the IR and Sham groups upon cluster analysis.

\section{Prediction of mRNAs of miRNAs and construction of the network}


The189 miDEGs were used to predict mRNAs in miRWalk 3.0.[9] Through filtering, 40 miRNAs (24 upregulated,16 down-regulated) were found to be associated with 240 predicted target genes (Table 2). The network (Fig. 3A) based on these 40 miRNAs and 240 mRNAs were constructed and visualized by Cytoscape.

\section{Gene Ontology Analyses of differentially expressed miRNAs}

Figure 3B presents the results of the $\mathrm{GO}$ analysis for the miRNAs that the number of mRNAs is $\geq 3$. The target genes(mRNAs) of downregulated mmu-miR-185-5p were mainly enriched in p53 binding, whereas the unregulated mRNAs of mmu-miR-7a-5p were significantly enriched in histone deacetylase binding, RNA polymerase II specific DNA-binding transcription factor binding, and DNA-binding transcription factor binding. The target genes of mmu-miR-106b-5p and mmu-miR-449a-5p were both enriched in repressing transcription binding. As shown in Fig. 3A,the two miRNAs were up-regulated. It is well known that miRNA can bind to target RNA transcripts, resulting in the repression of target gene expression. So, the function of transcription faction binding was activated, which corresponded to intestinal I/R injury.

\section{Construction of PPI network}

Based on the interactions retrieved from STRING, PPI network was constructed.

Between the 240 mRNAs, 60 mRNAs showed protein-protein interactions (Table 4), and 57 of them were interacted with 16 miRNAs in the above 40 miRNAs (Fig. 3A). In this network, most mRNA were targeted by mmu-miR-709, and there are interactions between its target genes.

\section{CeRNA regulating network}

The 40 miRNAs that matched mRNAs in the previous section were chosen to match IncRNAs to further highlight the link between IncRNAs and miRNAs. Upon a search, 26 miRNAs were found to be associated with IncRNAs, yielding 451 regulatory connections between miRNAs and IncRNAs (Table 5). MiRNAIncRNAs that were validated by more than 3 CLIP-seq experiments(clipExpNum $>3$ )were included in the Starbase. Eight up-regulated, and 2 down-regulated miRNAs are matched with 8 IncRNAs following filtration (Table 6, Fig. 3C), and the resulting CeRNA regulatory network was built using miRNA-mRNAs (Fig. 3D). Then, Cytoscape was used to visualize the interacting relationships between mRNA, miRNA and IncRNA, which is shown in Fig. 4B. It is worth noting that mmu-miR-709 which were targeted by most mRNAs matched with none of the IncRNAs in starBase by now, so it was not included in ceRNA networks.

\section{Validation of key genes in the ceRNA}

The hub miDEGs (Fig. 3D) identified by CytoHubba were selected to reduce the scope of ceRNA networks. As shown in this ceRNA network, IncRNA 1700020114Rik matched with miRNAs mmu-miR-363-3p and mmu-miR-7a-5p, while IncRNA Gm26917 matched with miRNAs mmu-miR-185-5p, mmu-miR-665-5p, and mmu-miR-339-5p. Then ceRNA networks were then used to derive four crucial IncRNA/miRNA/mRNA modules. GSE97633 dataset was applied to confirm the major genes in the ceRNA network of intestinal I/R injury depicted in the previous study. The same approach and criterion were employed to obtain the 
mDEGs from GSE97633. Moreover, the mDEGs from GSE97633 were intersected with the anticipated mRNAs associated with miDEGs in dataset GSE83701. The results show that the 4 genes, which are Lrrc58, Sdad1, Klf4 and Cd69, are the intersection genes. The terminal results are: IncRNA 1700020114Rik/mmu-miR-7a-5p/Klf4 and IncRNA Gm26917/mmu-miR-665-3p/ Lrrc58 network (Fig. 3D).

\section{Real-time Polymerase Chain Reaction (RT-PCR)}

H\&E staining and histological injury scoring (Chiu's score) of the intestinal mucosa were shown in Fig. 4A-C. The qRT-PCR was used to measure the expression of above ceRNAs. The expression of IncRNA 1700020114Rik and Klf4 decreased markedly $(p<0.05)$ in intestinal I/R injury, while the mmu-miR-7a-5p and Lrrc58 expression level increased, as shown in Fig. 4D-F and I. There was no statistical difference in the expression of IncRNA Gm26917 and miRNA mmu-miR-665-5p between the two groups (Fig. 4G-H). Finally, IncRNA 1700020114Rik/mmu-miR-7a-5p/Klf4 ceRNA network was constructed.

\section{Discussion}

LncRNA, regulates the expression of protein-coding genes via functioning as miRNA sponges. Numerous studies have elucidated that IncRNAs extensively participate in crucial physiological processes such as metabolism and immunity, and are closely linked to the emergence and progression of tumors, cardiovascular diseases, nervous system disorders, nephropathy, and other diseases[10]. However, the mechanism of IncRNA-mediated ceRNA in intestinal I/R injury remains unclear. In this study, the expression datasets of miRNA of intestinal I/R injury samples were collected from the NCBI GEO datasets GSE83701. LncRNA and mRNA were predicted by database through miDEGs, and IncRNA 1700020114Rik/mmu-miR-7a-5p/KIf4 ceRNA network was constructed by Cytoscape. The animal experiments validated that LncRNA 1700020I14Rik, Klf4 were downregulated. Meanwhile, miR-7a-5p was up-regulated in intestinal I/R injury. Besides,there has been no report of the $11700020114 \mathrm{Rik} / \mathrm{mmu}-\mathrm{miR}$ 7a-5p/KIf4 ceRNA network in intestinal I/R injury so far.

LncRNA1700020114Rik is also known as Oip5os1 and Oip5as1.Studies[11, 12] have elucidated that IncRNAs 1700020114Rik play a key role in apoptosis, cell proliferation and fibrosis, oxidative stress, and other physiological processes. In addition, other studies[11, 13] revealed that LncRNA 1700020114Rik was downregulated in the hearts of rats with myocardial I/R injury and hypoxia / reoxygenation( $H / R$ )injury in myocardial cells. Researchers discovered that sponging miR-186-5p with up-regulated IncRNA OIP5-AS1 reduced neuron damage in microglia/macrophages following middle cerebral artery occlusion/reperfusion produced inflammation and oxidative stress.[14]

The mmu-miR-7a-5p sequence in Mus musculus is a product of miR-7 genes. miR-7a-5p has controversial roles in multi-organ injury. Some researches $[15,16]$ found that miR-7a-5p may play a protective role in intestinal and brain. However, other studies demonstrated that miR-7a-5p was up-regulated during the cerebral I/R injury[17], acute lung injury[18], and myocardial injury induced by lipopolysaccharide [19]. The qRT-PCR results showed that miR-7a-5p was up-regulated after intestinal I/R injury. 
KIf4 can be regulated by miRNA at both transcriptional and post-transcriptional levels[20]. Klf4 is a multifunctional transcription factor that regulates diverse cellular processes in a context-dependent manner[20]. Thus, the role of Klf4 is determined by different genes interacting with Klf4. Hummitzsch et al.[21] found that the expression level of Klf4 was decreased in Human Regulatory Macrophages after $\mathrm{H} / \mathrm{R}$. Meanwhile, KIf4 could alleviate lipopolysaccharide-induced inflammation and cerebral vascular injury after cerebral ischemic stroke $[22,23]$. Therefore, Klf4 could help to alleviate I/R injury.

Overall, the IncRNA 1700020I14Rik, Klf4 and miR-7a-5p were associated with ischemia/reperfusion injury or oxygen-glucose deprivation injury.

In addition, IncRNA 1700020I14Rik and KIf4 were downregulated, and miR-7a-5p was up-regulated in ischemia/reperfusion injury in the ceRNA network identified by the bioinformatics analysis. This was in accordance with the experimental validation in intestinal I/R injury. Furthermore, studies[18] have confirmed that miR-7a-5p has interactions with Klf4 in acute lung injury.

Therefore, the $1700020114 \mathrm{Rik} / \mathrm{mmu}-\mathrm{miR}-7 \mathrm{a}-5 \mathrm{p} / \mathrm{Klf} 4$ axis was postulated to play a potential role in intestinal I/R injury. It was the first time to uncover IncRNA mediated ceRNA networks in intestinal I/R injury. However, the role of 1700020114 Rik/mmu-miR-7a-5p/KIf4 ceRNA network in intestinal I/R injury needs to be examined in further studies.

\section{Conclusion}

The findings provide a fresh insight at ceRNA regulation mechanism in intestinal I/R injury, identifying 170002700020114Rik/mmu-miR-7a-5p/Klf4 ceRNA network as potential targets for prevention and treatment of intestinal I/R injury.

\section{Materials And Methods}

\section{MiRNA and mRNA Microarray Data}

The GEO database, which is a gene expression database created and maintained by the National Center for Biotechnology Information (NCBI), stores curated gene expression datasets. The miRNA/mRNA expression profiles GSE96733[24] and GSE83701[8] from NCBI-GEO were used in mice intestinal I/R injury study.

\section{Differentially expressed miRNAs analysis}

The data was divided into two groups: the IR group and the Sham group. The limma package (Version 3.26.9)[25] and Bayes test were employed to identify the differentially expressed miRNAs/mRNAs (miDEGs and $\mathrm{mDEGs}$ ) between the sham samples and intestinal I/R injury samples. Multiple-test correction with Benjamini and Hochberg's method was performed for $p$-value calculation. Genes with $p$ value $<0.05$ and $|\log 2 \mathrm{FC}|>1.5$ were regarded as the thresholds of miDEGs or mDEGs. Gene with $p$-value 
$<0.05$ and $\log 2 \mathrm{FC}>1.5$ are defined as up-regulated miDEGs/mDEGs, and with $p$-value $<0.05$ and $\log 2 \mathrm{FC}<-1.5$ are defined as down-regulated miDEGs/mDEGs. The pheatmap package (version 1.0.12)[26] was applied for the bidirectional hierarchical clustering, and the expression values were presented using heatmaps.

\section{Predicting miRNA-mRNAs}

Their potential mRNAs that matched with miDEGs were predicted by miRWalk 3.0 [9]. In addition, target mRNAs were validated by experiments and simultaneously predicated by the miRDB or Targetscan databases.

\section{Protein-protein interaction(PPI) network generation and module analysis}

The STRING database [27] (version:11.0b,https://string-db.org)

was used to construct PPI network for target genes-encoded proteins, including direct and indirect associations. The Required Confidence (combined score) $>0.7$ [27] was set as threshold value. The Cytoscape was utilized to visualize the raw data of network from STRING database and then displayed [28](version 3.8.0, https://www.cytoscape.org). MiRNA nodes of every gene were added to PPI network to analyze the main regulatory mechanism of miRNAs.

\section{Gene Ontology Analyses of differentially expressed miRNAs}

Gene Ontology (GO) enrichment analysis was constructed as functional analysis of miDEG-targeted mRNA. The GO analysis was performed in R software with clusterProfiler package[29] and was visualized by ggplot2 package[30] in R Studio.

\section{Construction of ceRNA regulating network}

IncRNAs that target MiRNAs were searched using starBase Version [31] (Version 3.0,http:// starbase.sysu.edu.cn)

also called ENCORI. The interacting relationships were visualized via Cytoscape. The IncRNA-miRNA network and miRNA-mRNA genes network were combined to form IncRNA-miRNA-mRNA network, also known as the ceRNA network[32]. The hub IncRNA-miRNA-mRNA networks were selected by Cytoscape plug-in CytoHubba[33]. Finally, the critical ceRNA network of intestinal I/R injury was selected using mRNAs found in both GSE97633 mDEGs and the target mRNAs of GSE83701 miDEGs.

\section{Confirmation of RNA expression of ceRNA after intestinal ischemia/reperfusion injury}

\section{Animal Model}


Male C57BL/6(weighing 20-25g, 7-8w) mice were obtained from Nanfang Hospital, Southern Medical University, and then housed at separated cages in a temperature-controlled room under a fixed circadian rhythm with free access to food and water. The animal experiments were approved by the Ethics Committee of Nanfang Hospital (China, application No: NFYY-2021-54)and all procedures were carried out in compliance with National Institutes of Health guidelines for the use of experimental animals. According to a previous study, the intestinal I/R injury was generated by occluding the superior mesenteric artery (SMA) with a microvascular clamp for 45 mins followed by 240 mins of reperfusion. In addition, the Sham group was the same with intestinal I/R injury except occluding the SMA [8]'[34].

\section{Sample Collection and Histological Staining}

Intestine segments were collected at the end of reperfusion and then divided into two segments, based on past research [8]. One of the segments was dried with filter paper and preserved at $-80^{\circ} \mathrm{C}$, waiting for detection. The other was treated in $4 \%$ paraformaldehyde as previously described [35]. The intestinal injury score were measured by automated image analysis in five random 200X fields of each sample [36].

\section{Quantitative Reverse Transcription-Polymerase Chain Reaction (qRT-PCR) analysis}

Trizol reagent was used to extract total RNA according to the manufacturer's protocol. The mRNA and miRNA was reversed into cDNA by kits from Toyobo and Takara, according to the previous study[36].LncRNA and mRNA quantitative analysis was conducted with qRT-PCR using Toyobo SYBR Green Realtime PCR (Applied Biosystems, Foster City, CA), whereas the expression of miRNA was evaluated via Takara TB Green Advantage qPCR Premix (200rxn, Cat.No.639676) .18S and U6 served as internal controls, and the fold change relative to the control was computed by the $2^{-\Delta \Delta C t}$ method. Table 1 displyas the Forward and Reverse primer sequences.

\section{Statistical Analysis}

Statistical analyses were conducted using R (version 4.0.3) and GraphPad Prism version 8.0 (GraphPad Software, Inc., San Diego, CA, USA).

The results were provided as mean \pm standard error of the mean (SEM) from at least three independent replicates. Welch's t-test was used to estimate the differences between the groups. The statistically significant difference was defined as a $P$ value less than 0.05 .

\section{Declarations}

\section{Acknowledgement}

We appreciate Jianming Zeng's team's bioinformatics advice. We appreciate Bowei Zhou and Zhengzheng Yan for their advice in the writing of this manuscript. 


\section{Author Contributions}

Lin Zhu, Xiao Yang, Kexuan Liu and Bingcheng Zhao designed experiments, analyzed data and prepared this manuscript. Ziyi Wang,Yupei Lai and Shiting Xu performed animal experiments, ZhiWen Yao and Lin Zhu discussed the results, Kexuan Liu and Bingcheng Zhao revised this paper. All authors reviewed and approved the submitted the manuscript.

\section{Funding}

This work was supported by grants from the Key Program of National Natural Science Foundation, Beijing, China (81730058 to Ke-Xuan Liu).

\section{Availability of data and materials}

Data could be obtained upon request to the corresponding author.

\section{Ethics approval and consent to participate}

The animal experiments were approved by the Ethics Committee of Nanfang Hospital (China, application No: NFYY-2021-54). There are no clinical experiments in this study.

\section{Consent for publication}

Not applicable

\section{Conflict of interest}

The authors declare that they have no competing interests.

\section{References}

1. Kalogeris T, Baines CP, Krenz M, Korthuis RJ. Ischemia/Reperfusion Compr Physiol. 2016;7:113-70.

2. Ghafouri-Fard $S$, Shoorei $H$, Taheri M. Non-coding RNAs participate in the ischemia-reperfusion injury. Biomed Pharmacother. 2020;129:110419.

3. Mercer TR, Dinger ME, Mattick JS. Long non-coding RNAs: insights into functions. Nat Rev Genet. 2009;10:155-9.

4. Salmena L, Poliseno L, Tay Y, Kats L, Pandolfi PP. A ceRNA hypothesis: the Rosetta Stone of a hidden RNA. language? Cell. 2011;146:353-8. 
5. Ouyang M, Lu J, Ding Q, Qin T, Peng C, Guo Q. Knockdown of long non-coding RNA PVT1 protects human AC16 cardiomyocytes from hypoxia/reoxygenation-induced apoptosis and autophagy by regulating miR-186/Beclin-1 axis. Gene. 2020;754:144775.

6. Cao Y, Pan L, Zhang X, Guo W, Huang D. LncRNA SNHG3 promotes autophagy-induced neuronal cell apoptosis by acting as a ceRNA for miR-485 to up-regulate ATG7 expression. Metab Brain Dis. 2020;35:1361-9.

7. Xu M, Yang Y, Deng Q-W, Shen J-T, Liu W-F, Yang W-J, et al. Microarray Profiling and Functional Identification of LncRNA in Mice Intestinal Mucosa Following Intestinal Ischemia/Reperfusion. J Surg Res. 2021;258:389-404.

8. Feng D, Wang Z, Zhao Y, Li Y, Liu D, Chen Z, et al. circ-PRKCB acts as a ceRNA to regulate p66Shcmediated oxidative stress in intestinal ischemia/reperfusion. Theranostics. 2020;10:10680-96.

9. Dweep H, Gretz N, Sticht C. miRWalk database for miRNA-target interactions. Methods Mol Biol. 2014;1182:289-305.

10. Y C. Z L, X C, S Z. Long non-coding RNAs: From disease code to drug role. Acta pharmaceutica Sinica B. 2021;11. doi:10.1016/j.apsb.2020.10.001.

11. Hu F, Yang J, Chen X, Shen Y, Chen K, Fu X, et al. LncRNA 1700020114Rik/miR-297a/CGRP axis suppresses myocardial cell apoptosis in myocardial ischemia-reperfusion injury. Mol Immunol. 2020;122:54-61.

12. Ghafouri-Fard S, Dashti S, Farsi M, Hussen BM, Taheri M. A review on the role of oncogenic IncRNA OIP5-AS1 in human malignancies. Biomed Pharmacother. 2021;137:111366.

13. Wang J, Tang Q, Lu L, Luo Z, Li W, Lu Y, et al. LncRNA OIP5-AS1 interacts with miR-363-3p to contribute to hepatocellular carcinoma progression through up-regulation of SOX4. Gene Ther. 2019;27:495-504.

14. Chen Y, Liu W, Chen M, Sun Q, Chen H, Li Y. Up-regulating IncRNA OIP5-AS1 protects neuron injury against cerebral hypoxia-ischemia induced inflammation and oxidative stress in microglia/macrophage through activating CTRP3 via sponging miR-186-5p. Int Immunopharmacol. 2021;92:107339.

15. Singh AP, Hung $Y-H$, Shanahan MT, Kanke M, Bonfini A, Dame MK, et al. Enteroendocrine Progenitor Cell-Enriched miR-7 Regulates Intestinal Epithelial Proliferation in an Xiap-Dependent Manner. Cell Mol Gastroenterol Hepatol. 2020;9:447-64.

16. Kim T, Mehta SL, Morris-Blanco KC, Chokkalla AK, Chelluboina B, Lopez M, et al. The microRNA miR7a-5p ameliorates ischemic brain damage by repressing a-synuclein. Sci Signal. 2018;11.

17. Zhao J, Wang B. MiR-7-5p Enhances Cerebral Ischemia-Reperfusion Injury by Degrading sirt1 mRNA. J Cardiovasc Pharmacol. 2020;76:227-36.

18. Zhao J, Chen C, Guo M, Tao Y, Cui P, Zhou Y, et al. MicroRNA-7 Deficiency Ameliorates the Pathologies of Acute Lung Injury through Elevating KLF4. Front Immunol. 2016;7:389.

19. Liang D, Jin Y, Lin M, Xia X, Chen X, Huang A. Down-regulation of Xist and Mir-7a-5p improves LPSinduced myocardial injury. Int J Med Sci. 2020;17:2570-7. 
20. Ghaleb AM, Yang VW. Krüppel-like factor 4 (KLF4): What we currently know. Gene. 2017;611:27-37.

21. Hummitzsch L, Zitta K, Rusch R, Cremer J, Steinfath M, Gross J, et al. Characterization of the Angiogenic Potential of Human Regulatory Macrophages (Mreg) after Ischemia/Reperfusion Injury In Vitro. Stem Cells Int. 2019;2019:3725863.

22. Li Z, Jia Y, Han S, Wang X, Han F, Zhang J, et al. KIf4 Alleviates Lipopolysaccharide-Induced Inflammation by Inducing Expression of MCP-1 Induced Protein 1 to Deubiquitinate TRAF6. Cell Physiol Biochem. 2018;47:2278-90.

23. Zhang X, Wang L, Han Z, Dong J, Pang D, Fu Y, et al. KLF4 alleviates cerebral vascular injury by ameliorating vascular endothelial inflammation and regulating tight junction protein expression following ischemic stroke. J Neuroinflammation. 2020;17:107.

24. Karhausen J, Bernstock JD, Johnson KR, Sheng H, Ma Q, Shen Y, et al. Ubc9 overexpression and SUM01 deficiency blunt inflammation after intestinal ischemia/reperfusion. Lab Invest. 2018;98:799-813.

25. Phipson B, Lee S, Majewski IJ, Alexander WS, Smyth GK. ROBUST HYPERPARAMETER ESTIMATION PROTECTS AGAINST HYPERVARIABLE GENES AND IMPROVES POWER TO DETECT DIFFERENTIAL EXPRESSION. Ann Appl Stat. 2016;10:946-63.

26. Raivo K, pheatmap: Pretty Heatmaps. R package version 1.0.12. 2019. https://CRAN.Rproject.org/package=pheatmap.

27. von Mering C, Huynen M, Jaeggi D, Schmidt S, Bork P, Snel B. STRING: a database of predicted functional associations between proteins. Nucleic Acids Res. 2003;31:258-61.

28. Shannon P, Markiel A, Ozier O, Baliga NS, Wang JT, Ramage D, et al. Cytoscape: a software environment for integrated models of biomolecular interaction networks. Genome Res. 2003;13:2498-504.

29. Yu G, Wang L-G, Han Y, He Q-Y. clusterProfiler: an R package for comparing biological themes among gene clusters. OMICS. 2012;16:284-7.

30. Wickham H. ggplot2: Elegant Graphics for Data Analysis. 2016. https://ggplot2.tidyverse.org.

31. Li J-H, Liu S, Zhou H, Qu L-H, Yang J-H. starBase v2.0: decoding miRNA-ceRNA, miRNA-ncRNA and protein-RNA interaction networks from large-scale CLIP-Seq data. Nucleic Acids Res. 2014;42:92-7. Database issue:D.

32. Yao K, Yu Y, Zhang H. Construction for Long Non-Coding RNA (IncRNA)-Associated Competing Endogenous RNA (ceRNA) Network in Human Retinal Detachment (RD) with Proliferative Vitreoretinopathy (PVR). Med Sci Monit. 2020;26. doi:10.12659/MSM.919871.

33. Chin $\mathrm{C}-\mathrm{H}$, Chen $\mathrm{S}-\mathrm{H}, \mathrm{Wu} \mathrm{H}-\mathrm{H}, \mathrm{Ho} \mathrm{C}-\mathrm{W}, \mathrm{Ko}$ M-T, Lin C-Y. cytoHubba: identifying hub objects and subnetworks from complex interactome. BMC Syst Biol. 2014;8(Suppl 4):11.

34. Deng F, Hu J, Yang X, Wang Y, Lin Z, Sun Q, et al. Interleukin-10 expands transit-amplifying cells while depleting Lgr5 + stem cells via inhibition of Wnt and notch signaling. Biochem Biophys Res Commun. 2020;533:1330-7. 
35. Deng F, Zhao B-C, Yang X, Lin Z-B, Sun Q-S, Wang Y-F, et al. The gut microbiota metabolite capsiate promotes Gpx4 expression by activating TRPV1 to inhibit intestinal ischemia reperfusion-induced ferroptosis. Gut Microbes. 2021;13:1-21.

36. Zhou B, Zhang W, Yan Z, Zhao B, Zhao J, Feng W, et al. MicroRNA-26b-5p Targets DAPK1 to Reduce Intestinal Ischemia/Reperfusion Injury via Inhibition of Intestinal Mucosal Cell Apoptosis. Dig Dis Sci. 2021. doi:10.1007/s10620-021-06975-7.

\section{Tables}

Due to technical limitations, tables are only available as a download in the Supplemental Files section.

\section{Figures}




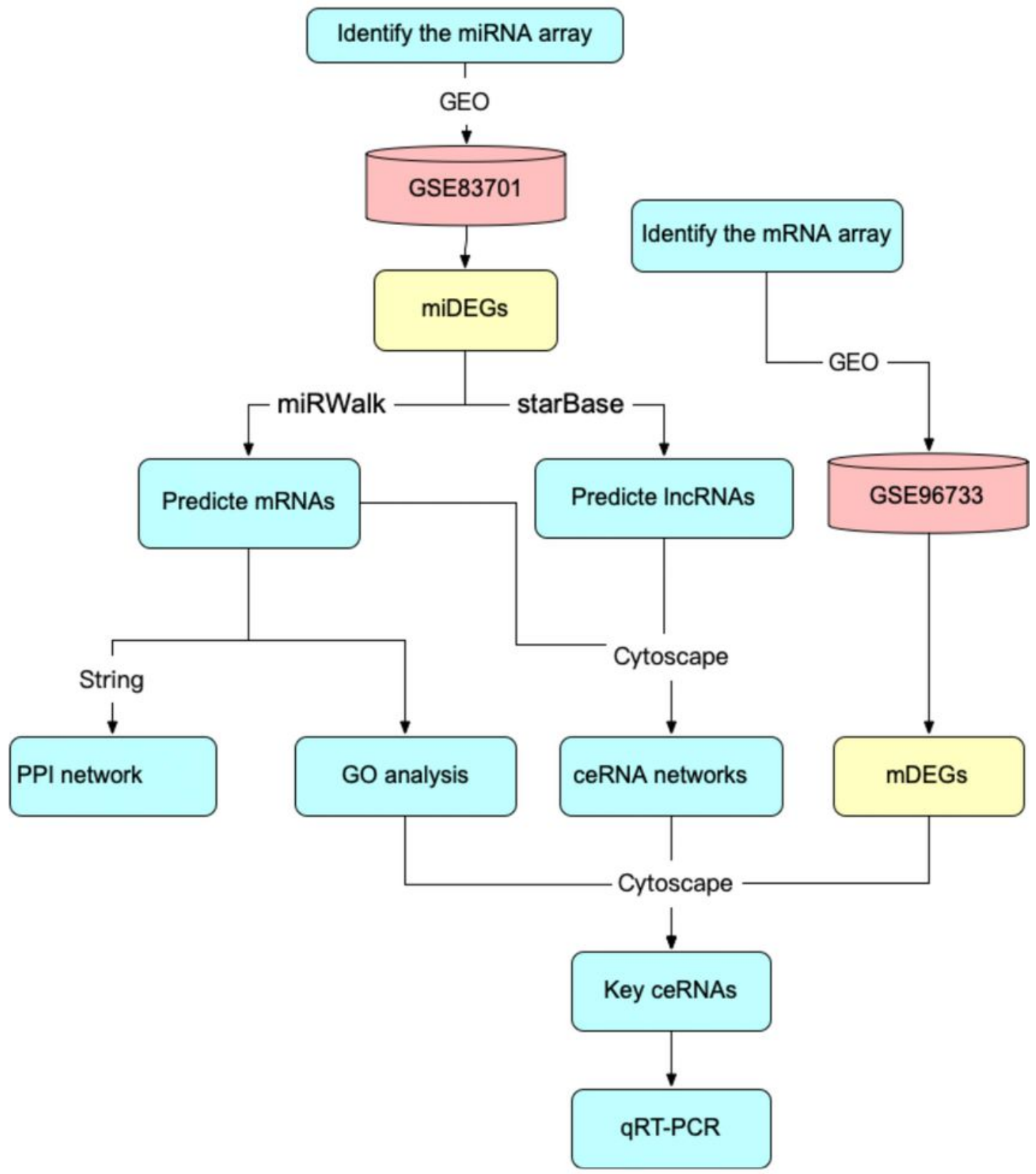

Figure 1

The flow chart of the study. 


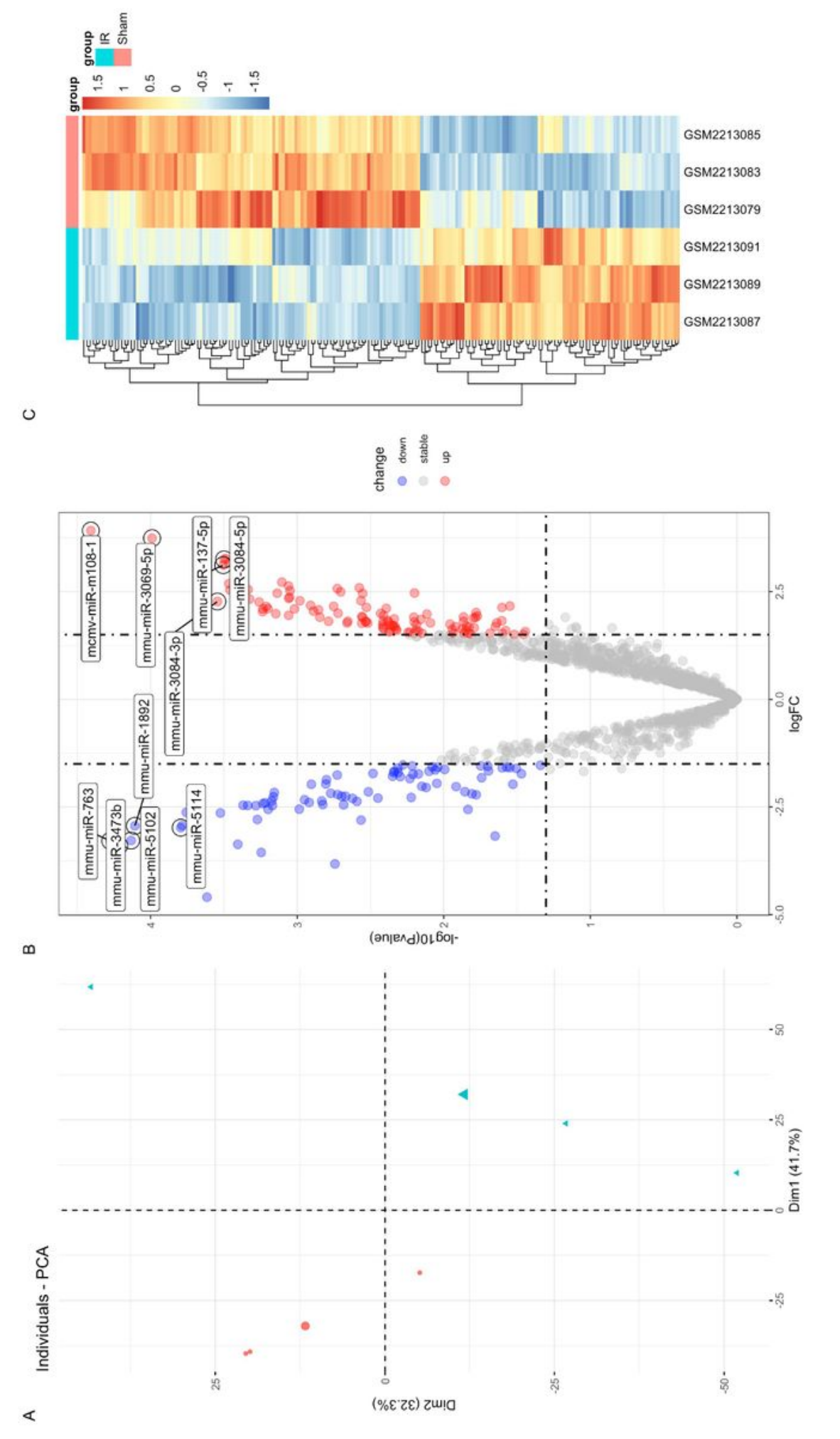

\section{Figure 2}

Differentially expressed miRNAs: (A) The PCA plot. (B) In the volcano plot of miDEGs, the horizontal axis represents the fold change (IR versus Sham), and the vertical axis represents the p-value of miDEGs, genes with the absolute value of |log2FC |>1.5 and p-value. (C) In the heat map of miDEGs, the horizontal axis represents the name of each sample, while the left vertical axis represents the degree of gene clustering. Red represented the up-regulated genes, while blue stands for the downregulated genes. 
A

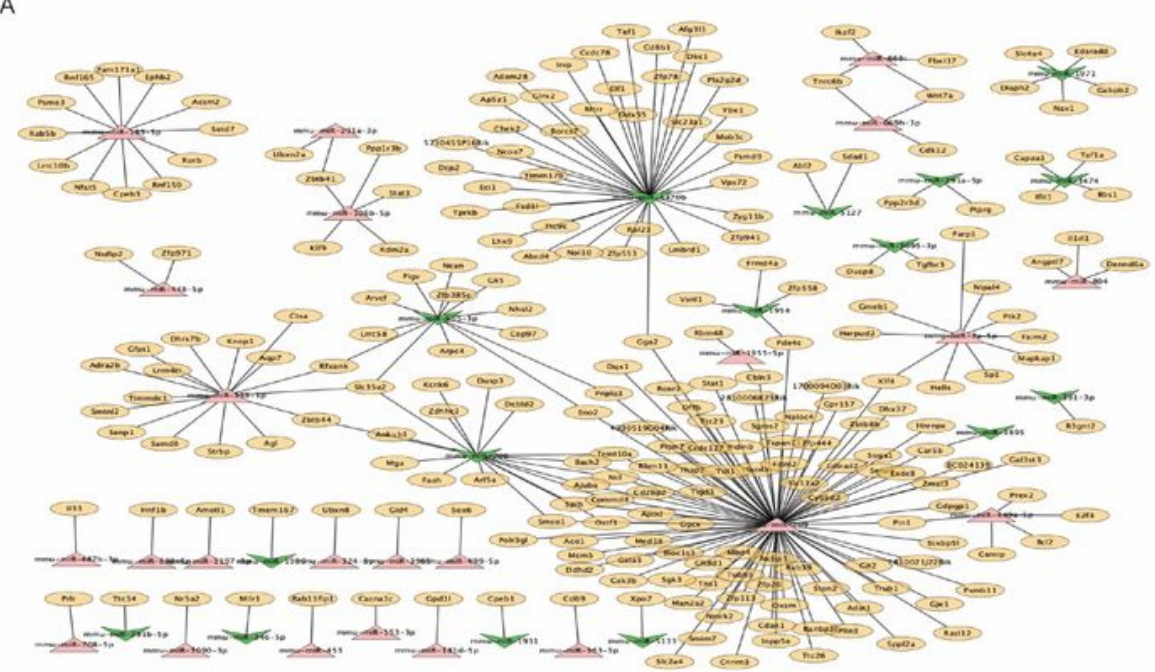

B

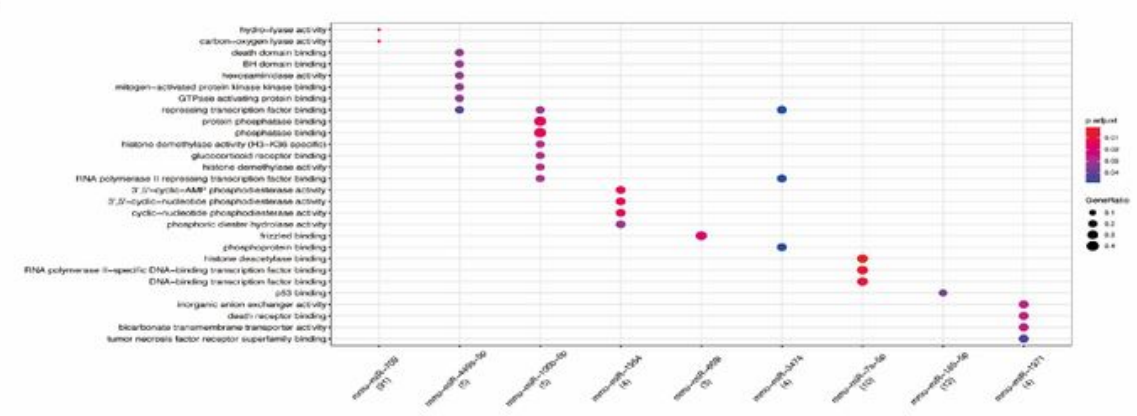

C

D
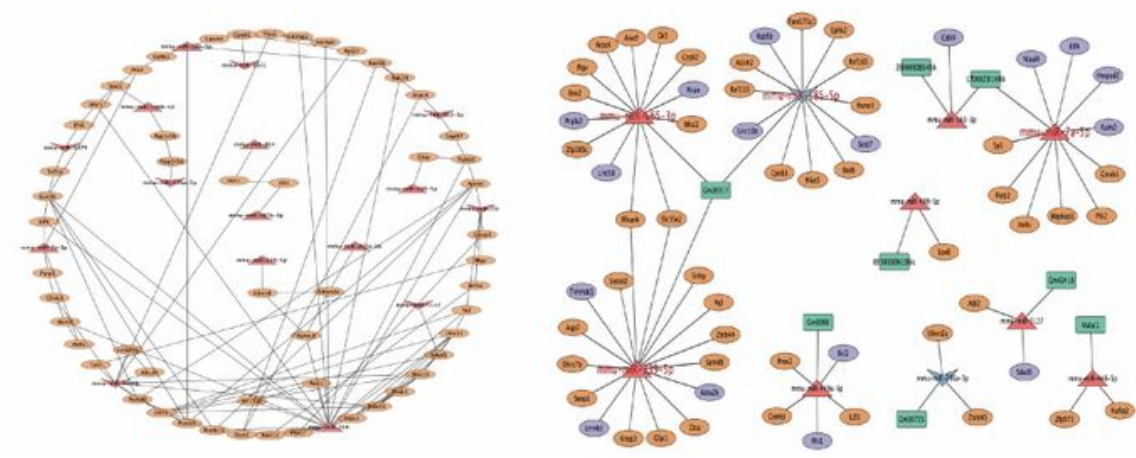

\section{Figure 3}

The miRNA-mRNA network, GO analysis, PPI and ceRNA networks. (A)The miRNA-mRNA network. The network was based on these 40 miRNAs and 240 target genes. Yellow circle nodes: target genes; red triangle: up-regulated miRNAs; grey arrow: down-regulated miRNAs. (B)The result of GO analyses for the miRNAs. The lateral axis: miRNAs, numbers in parentheses: the number of genes; vertical axis: the name of GO terms; the size of round dot: GeneRatio, the bigger dot represents more miRNAs enriched in this 
term, the smaller p-value represents higher significance. (C)Protein-protein interaction (PPI)networks. Orange circle nodes: mRNAs; red triangle: up-regulated miRNAs; green arrow: down-regulated miRNAs; line without arrow: PPI relations; line with an arrow: miRNA-gene regulating relations. (D)Competing endogenous RNAs (ceRNA) regulating networks. Yellow circle nodes: target mRNAs囚purple circle nodes: intersected target mRNAs between two datasets; red cycle: IncRNA; green arrow: down-regulated miRNAs; red triangle: up-regulated miRNAs, miRNA with red label were hub miRNAs chosen by cytoHubba.

A

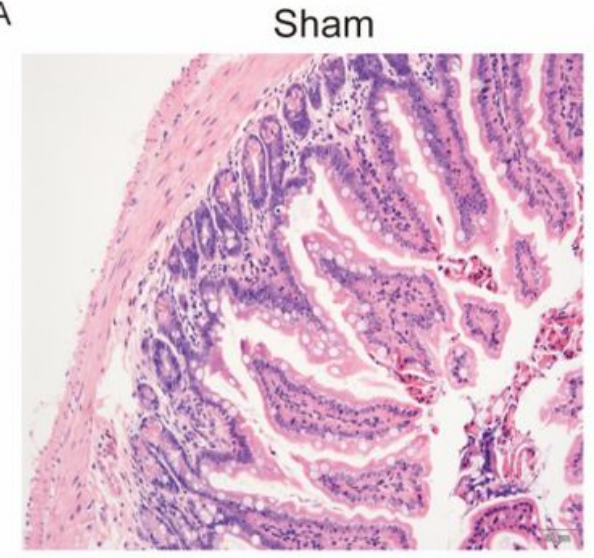

D

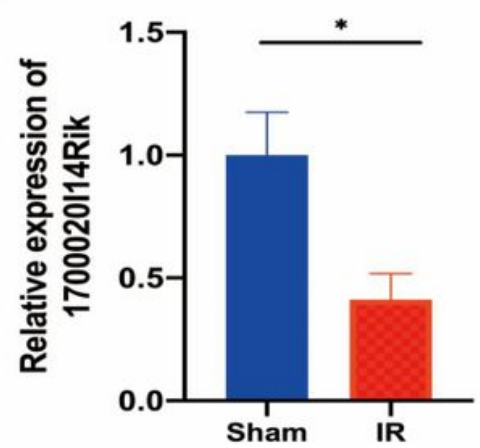

G

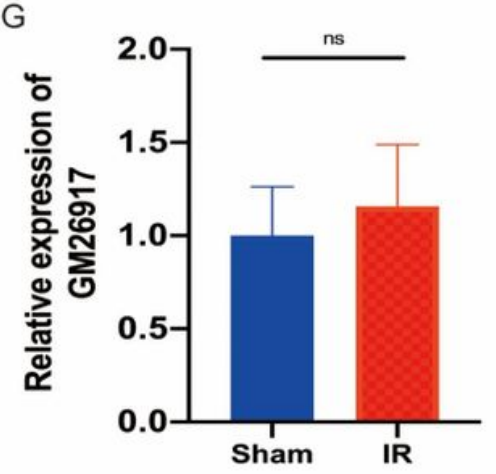

B

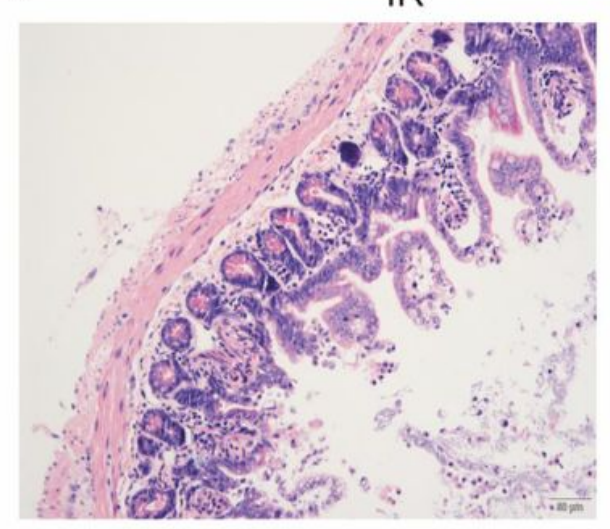

$\mathrm{E}$

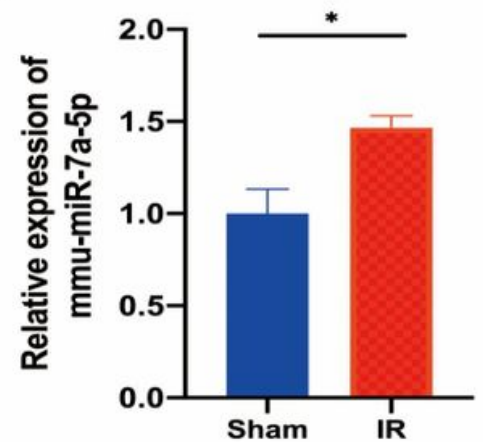

$\mathrm{H}$

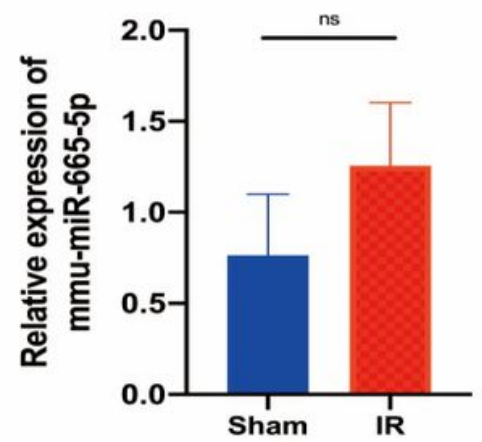

C

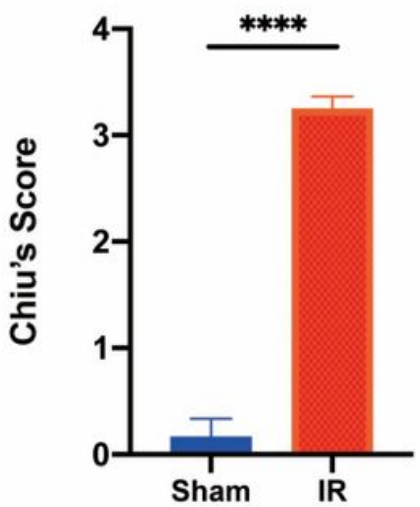

$\mathrm{F}$
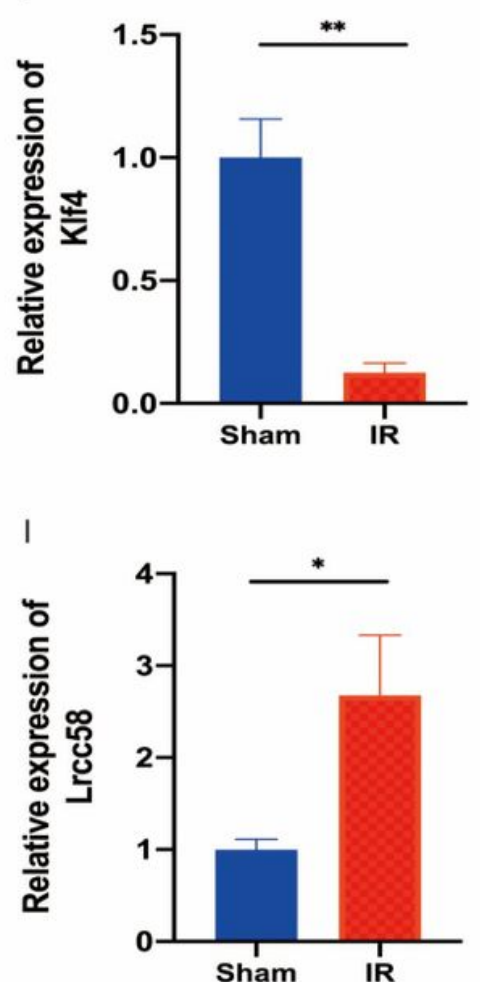

Figure 4

Histological injury scoring and qRT-PCR results of the small intestine. (A-B) H\&E staining and histological injury scoring (Chiu's score) of the intestinal mucosa. Scale bar, $50 \mu m(n=5,6)$. (C-H) Relative expression 
of the IncRNA/miRNA/mRNA by qRT-PCR $(n=4-6)$. The results are expressed as the mean \pm SEM. * $p<$ $0.05,{ }^{* *} p<0.01,{ }^{* \star *} p<0.001 \nabla^{\star \star \star *} p<0.001$ by two-tailed Welch's t-test.

\section{Supplementary Files}

This is a list of supplementary files associated with this preprint. Click to download.

- ZhaoSupplymentaryFigure1.docx

- ZhaoSupplymentaryTables.xlsx 